\title{
Microheterogeneity of Topoisomerase IA/IB and Their DNA-Bound States
}

\author{
Kevin Jeanne Dit Fouque, ${ }^{\dagger, \|}$ Alyssa Garabedian, ${ }^{\dagger, \|}$ Fenfei Leng, ${ }^{\dagger, \|}$ Yuk-Ching Tse-Dinh, ${ }^{\dagger, \|}$ \\ and Francisco Fernandez-Lima* ${ }^{*},, \|_{\odot}$
}

${ }^{\dagger}$ Department of Chemistry and Biochemistry and "Biomolecular Sciences Institute,Florida International University, 11200 SW 8th St., AHC4-233, Miami, Florida 33199, United States

Supporting Information

ABSTRACT: Topoisomerases are important complex enzymes that modulate DNA topology to maintain chromosome superstructure and integrity. These enzymes are involved in many cellular processes that resolve specific DNA superstructures and intermediates. The low abundance combined with the biological heterogeneity of relevant intermediates of topoisomerases makes their structural information not readily accessible using traditional structural biology tools (e.g., NMR and X-ray crystallography). In the present work, a secondgeneration trapped ion mobility spectrometry-mass spectrometry (TIMS-MS) was used to study Escherichia coli topoisomerase IA (EcTopIA) and variola virus topoisomerase IB (vTopIB) as well as their complexes with a single-stranded

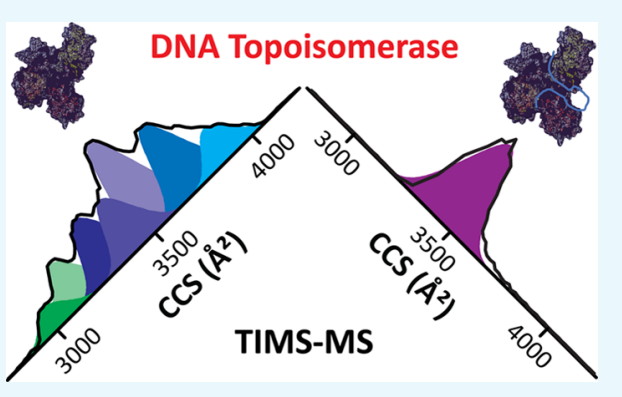
DNA and a stem-loop DNA under native conditions. The higher trapping efficiency and extended mass range of the new, convex TIMS geometry allowed for the separation and identification of multiple conformational states for the two topoisomerases and their DNA complexes. Inspection of the conformational space of EcTopIA and vTopIB in complex with DNA showed that upon DNA binding, the number of conformational states is significantly reduced, suggesting that the DNA binding selects for a narrow range of conformers restricted by the interaction with the DNA substrate. The large microheterogeneity observed for the two DNA binding proteins suggests that they can have multiple biological functions. This work highlights the potential of TIMS-MS for the structural investigations of intrinsically disordered proteins (e.g., DNA binding proteins) as a way to gain a better understanding of the mechanisms involved in DNA substrate recognition, binding, and assembly of the catalytically active enzyme-DNA complex.

\section{INTRODUCTION}

DNA topoisomerases are ubiquitous enzymes present in every organism that manage and control DNA topology by releasing torsional stress from supercoiled DNA during replication and transcription. ${ }^{1}$ The level of DNA supercoiling has substantial implications in essential cellular functions, making the study of topoisomerases and DNA topological problems during various cellular transactions of great biological importance. ${ }^{2,3}$ Previous work has also shown bacterial topoisomerase I as an attractive antibiotic drug target because of their importance in bacterial growth and low homology with other human topoisomerases. ${ }^{4}$ Moreover, the unlinking of DNA strands by topoisomerases is not a single-step process; it involves multiple intermediates. ${ }^{5} \mathrm{~A}$ main roadblock of the investigation of these intermediates is the lack of a spectrum of relevant three-dimensional structures for the topoisomerases and their DNA-bound forms. Under the protein folding problem, ${ }^{6}$ proteins do not necessarily have a unique ground state (folded state or native structure) representing a single free-energy minimum but rather a distribution of ground states with the same energy. This is typically known as a conformational space or distribution, where each conformation can be described as a microstate. The distribution of microstates is related to the protein function. Protein "free-energy conformational landscape" can be extremely rugged; proteins might often be found in metastable states (i.e., partial energy minima low-lying state), where they are unable to reach the true ground state without climbing over a large energy barrier.

The biological heterogeneity of conformations for topoisomerases and their DNA-bound forms makes their structural information not readily accessible using traditional structural biology tools, such as nuclear magnetic resonance (NMR) and X-ray crystallography. In the past, these techniques have been limited to the description of subdomains or DNA-bound subdomains. ${ }^{7-10}$ Hence, the development of alternative analytical tools able to describe the biological heterogeneity of conformations for topoisomerases and their DNA-bound forms is essential for investigating their multistep mechanisms consisting of DNA binding, cleavage, and strand religation.

Native mass spectrometry (MS), and more recently, in combination with ion mobility spectrometry (IMS), can provide unique insights in structural biology for the description of kinetic intermediates of biomolecules. ${ }^{1-14}$ The IMS-MS

Received: October 20, 2018

Accepted: January 11, 2019

Published: February 18, 2019 
coupling provides two dimensional separations: (i) separation based on the size/charge and (ii) separation based on their $\mathrm{m} /$ $z$. IMS-MS has addressed several shortcomings of conventional methods by reducing the analysis time (millisecond timescale), sample consumption (ng), and sample purity requirements. In addition, the recent implementation of trapped IMS (TIMS $)^{15,16}$ has shown better potential as compared to other IMS technologies for the separation and structural elucidation of biomolecules by providing high mobility resolving power ( $R$ up to 400 ) in short time scales (50-500 $\mathrm{ms}) .{ }^{17-24}$ Due to the higher trapping efficiency and mobility resolution advantages, TIMS-MS has gained significant attention for the investigation of proteins ${ }^{25-29}$ and $\mathrm{DNA}^{30,31}$ dynamics in their native and denatured states. When complemented with molecular dynamic simulations, IMSMS allows for the identification of specific intramolecular interactions that stabilize the kinetic intermediates. ${ }^{32,33}$ Moreover, a recent study using TIMS exhibited great promise for the structural investigation of peptide binding DNA under native conditions. ${ }^{34}$

The higher trapping efficiency and extended mass range provided by the new, convex TIMS geometry enabled, for the first time, the inspections of the conformational space of two intrinsically disordered DNA topoisomerases and their DNAbound states. In particular, we describe the conformational space of Escherichia coli topoisomerase IA (EcTopIA, Figure S1a) and variola virus topoisomerase IB (vTopIB, Figure S1b) and in complex with a single-stranded DNA and with a stemloop DNA. In the following discussion, special emphasis is placed on the capability to resolve microstates by the highmobility-resolution TIMS, expected from the flexible nature of topoisomerases IA and IB as well as the effect of the DNA binding on the overall topology of both topoisomerases.

\section{RESULTS AND DISCUSSION}

Topoisomerases can assume a variety of conformations to regulate DNA supercoiling. The proposed action for type IA and IB topoisomerases exhibits very different modes of operation. For example, topoisomerase IA uses a strandpassage mechanism, in which a single supercoil is removed per catalytic cycle, resulting in multiple topoisomer intermediates that lead to a much more controlled topological change. ${ }^{35}$ For topoisomerase IB, a free-rotation mechanism is used, where multiple supercoils are removed per catalytic cycle, resulting in a much lower number of topoisomer intermediates as compared to the strand-passage mechanism. ${ }^{36}$ However, structural information regarding the dynamics of topoisomerases when processing DNA supercoils is limited because of the inherent flexibility of the domains. A discussion on the microheterogeneity of EcTopIA and vTopIB is provided, and their measured collision cross sections (CCSs) are listed in Tables S1 and S2.

2.1. Native nESI-TIMS-MS Analysis of EcTopIA and DNA-Bound Form. The analysis of the EcTopIA enzyme $(97.5 \mathrm{kDa})$ under native conditions resulted in a narrow charge state distribution $(16+$ to $21+$, Figure $1 \mathrm{a}$ ) at $\mathrm{m} / \mathrm{z}$ of $4600-$ 6200 with multiple IMS bands per charge state (Figure $1 \mathrm{~b}$ ), corresponding to the intact EcTopIA native-like states. A pattern of conformational change was observed under native conditions, covering a CCS range of $\sim 5150$ to $6150 \AA^{2}$ (Table S1). Slight opening of the enzyme followed by conformational changes back to a more compact structure was observed with the increase of the charge state (Figure 1b). Moreover, these

\section{E. coli Topoisomerase IA (97.5 kDa)}
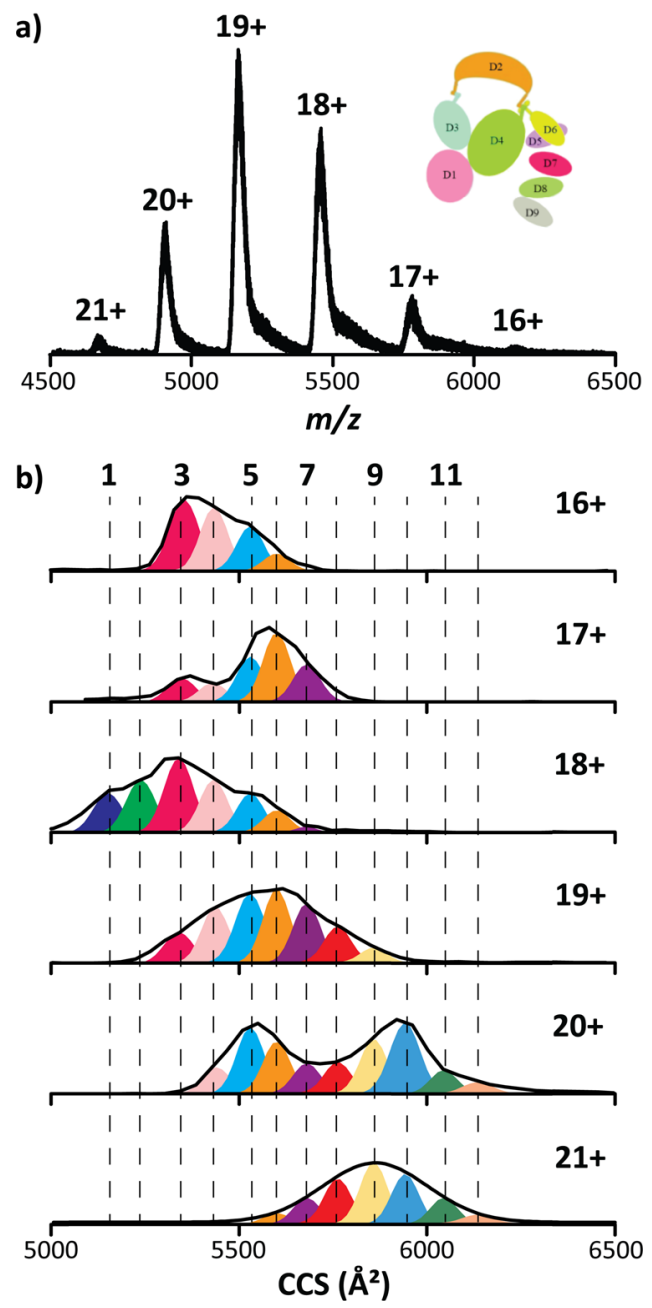

Figure 1. Typical MS (a) and TIMS (b) spectra for the multiply protonated species of E. coli topoisomerase IA. The dashed lines highlight the different microstates observed. A reported representation of the three-dimensional structure is shown in the inset for illustrative purposes.

changes were significant enough to identify 12 transition states (Figure 1b). This peculiar trend of opening and closing can give insight into the intermediates involved in the strandpassage mechanism and inter/intramolecular interactions that stabilize EcTopIA. In addition, the flexible nature of EcTopIA is evidenced by the large diversity of conformers. The results provided by native TIMS-MS data are consistent with previous structural studies performed on EcTopIA, ${ }^{37}$ supporting the presence of multiple structural microstates for type IA topoisomerases when not bound to DNA. Crystallographically independent structures of the $30 \mathrm{kDa}$ fragment of EcTopIA demonstrated that the two domains (D2, D3) present in this $30 \mathrm{kDa}$ fragment remain largely unchanged, and their relative arrangement can change dramatically. ${ }^{38}$ Spectroscopic characterization of EcTopIA in solution also showed extensive interdomain interactions involving both the N-terminal (D1D4) and C-terminal (D5-D9) domains. ${ }^{39}$ This indicates that the overall arrangement of the enzyme domains can change in a dramatic way. For example, the hinge between domains D2 and D4 may regulate the opening and closing of the $\mathrm{N}$ terminal domains. 
Structural analysis by means of traditional techniques often proves to be difficult because of the inherent flexibility of EcTopIA. However, a recent study reported a crystal structure of EcTopIA in complex with a single-stranded DNA, providing important insights into the mechanism of genome regulation by type IA topoisomerases responsible for removing negative DNA supercoils. ${ }^{10}$ Flexible linkers connect between some of the C-terminal domains. The large number of EcTopIA structural microstates may have been a factor as to why the crystal structure of full length EcTopIA could not be obtained until movements of the $\mathrm{C}$-terminal domains were restricted by the binding of the single-stranded DNA substrate.

The analysis of the E. coli topoisomerase I when in complex with a single-stranded DNA (105 kDa) under native conditions exhibited a 1:2 binding stoichiometry, resulting in four charge states $(17+$ to $20+$, Figure $2 a)$. It should be mentioned that the $1: 1$ stoichiometry complex was not observed. Inspection of the IMS profiles displayed similar CCS values when increasing the charge state (Figure 2b). DNA binding as observed in the crystal structure of full length E. coli topoisomerase I bound to single-stranded DNA (PDB
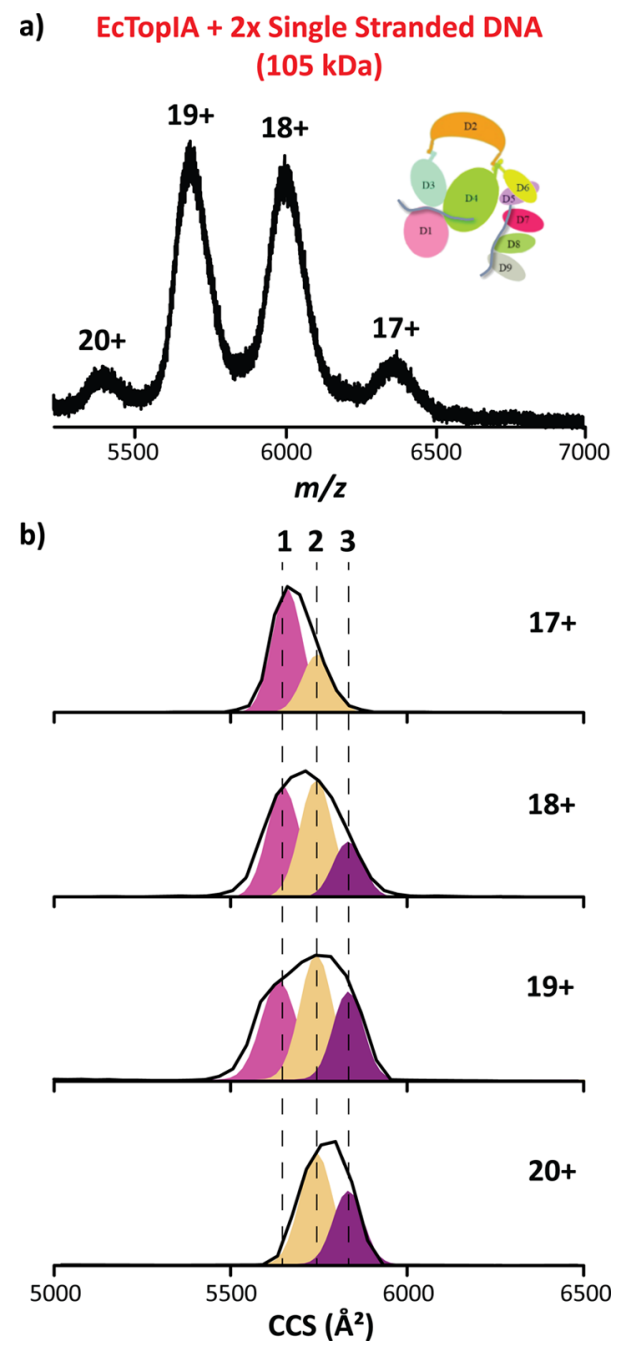

Figure 2. Typical (a) MS and (b) TIMS spectra for the multiply protonated species of E. coli topoisomerase IA in complex with two single-stranded DNAs. The dashed lines highlight the different microstates observed. A schematic of the DNA complex is represented in the inset for illustrative purposes.
4RUL) would select for a narrow range of conformers with relative orientations of the C-terminal domains (D5-D9) restricted by the interaction with the single-stranded DNA substrate. The comparison of the CCS range between EcTopIA and in complex with two single-stranded DNAs showed a smaller CCS range for the DNA-bound form binding $\left(150 \AA^{2}\right)$ when compared with the EcTopIA $\left(800 \AA^{2}\right)$ over the same native charge states, resulting in the identification of three microstates. In addition, slightly narrower mobility bands as compared to EcTopIA were observed, suggesting that the two single-stranded DNAs reduce the flexibility of the enzyme (Figure 2b).

The structural investigation of EcTopIA in complex with a stem-loop DNA (115 kDa) was also performed. The analysis of this DNA-bound complex under native conditions showed a $1: 1$ binding stoichiometry resulting in six charge states $(17+$ to $22+$, Figure $3 a)$. Inspection of the corresponding conformers exhibited narrower mobility bands as compared to EcTopIA and the DNA-bound form using the two single-stranded DNAs

a) EcTopIA + Stem Loop DNA (115 kDa)
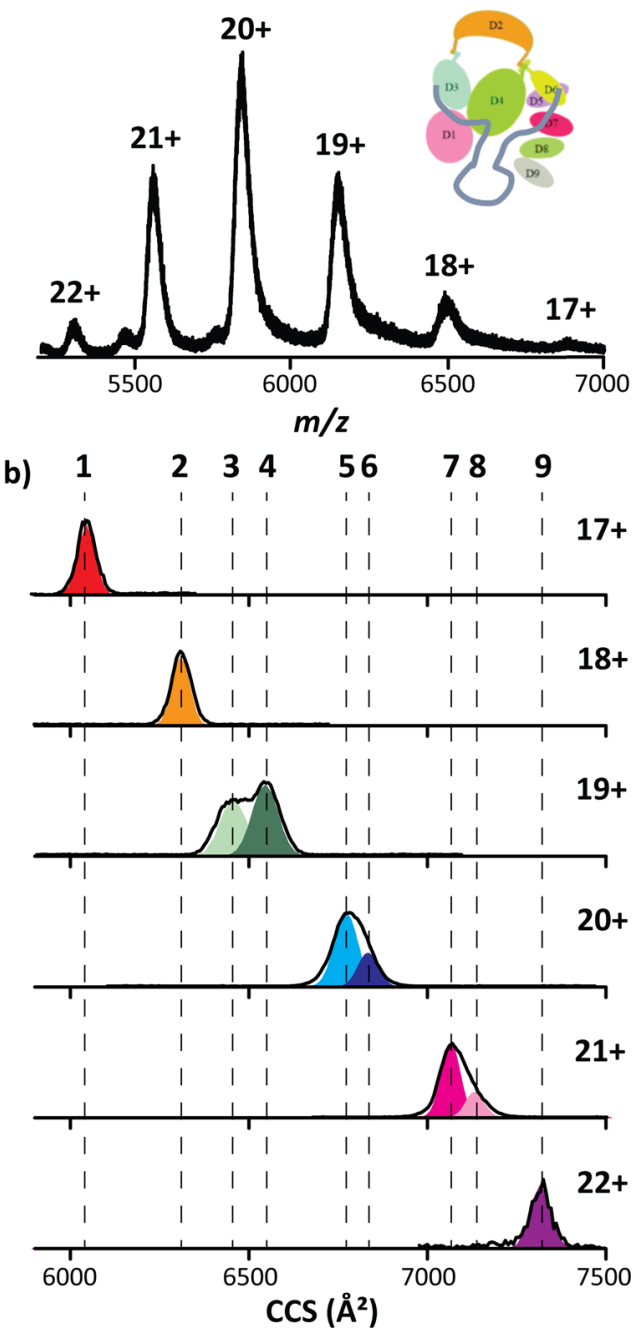

Figure 3. Typical (a) MS and (b) TIMS spectra for the multiply protonated species of $E$. coli topoisomerase IA in complex with a stem loop DNA. The dashed lines highlight the different microstates observed. A schematic of the DNA complex is represented in the inset for illustrative purposes. 
(Figure 3b). This suggests that the stem-loop DNA highly reduces the flexibility of the enzyme. In addition, a significant increase in the CCS values was observed per charge state, suggesting that multiple microstates can be obtained (Figure $3 \mathrm{~b})$. Comparison of the CCS range between EcTopIA in complex with two single-stranded DNAs and in complex with a stem-loop DNA showed a much larger CCS range in the case of the stem-loop DNA $\left(750 \AA^{2}\right)$ compared to the two singlestranded DNAs $\left(150 \AA^{2}\right)$ for the same charge states. Although a significant reduction of the flexibility of EcTopIA when bound to the stem-loop DNA was observed, several conformations were observed under native conditions, covering a CCS range of $\sim 5150$ to $6150 \AA^{2}$ (Table S1). The presence of more extended structures with the charge state is reflected by the significantly higher number of microstates for the stem-loop DNA binding (9) when compared to the DNAbound form with two single-stranded DNAs (3). In addition, conformational changes were observed, where more compact structures for the $19+$ charge state as well as an increase of the mobility bands for the $20+$ and $21+$ protonated species are obtained. This trend is different from the EcTopIA in the DNA-bound form with single-stranded DNA, suggesting that a larger number of intermediates are involved in the strandpassage mechanism and inter/intramolecular interactions that stabilize EcTopIA but could also be explained by a charge arrangement that more readily causes a structural transformation in the gas phase.

The stem-loop DNA is expected to interact with both the Nterminal and C-terminal domains of EcTopIA. The proteinDNA interactions can be observed readily in the available crystal structures (PDB 1MW8 for $\mathrm{N}$-terminal domain interactions and PDB 4RUL for C-terminal domain interactions) and studied in biochemical experiments. ${ }^{40}$ These include cation-phosphate and cation $-\pi$ interactions. Thus, binding of the stem-loop DNA would stabilize the charge states.

In addition, DNA cleavage may occur during incubation of topoisomerase with the substrate, especially for EcTopIA because the single-stranded DNA and stem-loop DNA used in this study contains a preferred cleavage site for this enzyme. Unlike negatively supercoiled duplex DNA, the single-stranded DNA and stem-loop DNA substrates do not become relaxed by the enzyme over the time course of incubation. DNA-bound EcTopIA complexes correspond to a mixture of ligated, cleaved/closed, and cleaved/open complexes, as described in the recent single-molecule study of topoisomerase IA gate dynamics. ${ }^{41}$ Although there is heterogeneity from the ligated versus cleaved state of DNA, single-stranded DNA and stemloop DNA binding reduce structural heterogeneity by limiting the large number of possible relative domain movements.

2.2. Native nESI-TIMS-MS Analysis of vTopIB and the DNA Bound Form. The analysis of the vTopIB enzyme $(38.5 \mathrm{kDa})$ under native conditions resulted in a narrow charge state distribution $(10+$ to $14+)$ at $m / z 2700-3900$ (Figure $4 a$ ). Inspection of the TIMS profiles showed the presence of several conformations, covering a CCS range of 2900 to $3950 \AA^{2}$ (Table S2). An opening of the enzyme is observed for the $13+$ protonated species, evidencing nine microstates (Figure $4 \mathrm{~b}$ ). The TIMS spectra displayed the native-like states for the $10+$ to $13+$ protonated species of vTopIB. A transition phase was observed starting at the +13 protonated species toward more open conformational states. The number of conformers provides insights into the number of transitional intermediates a) Variola virus Topoisomerase IB (38.5 kDa)

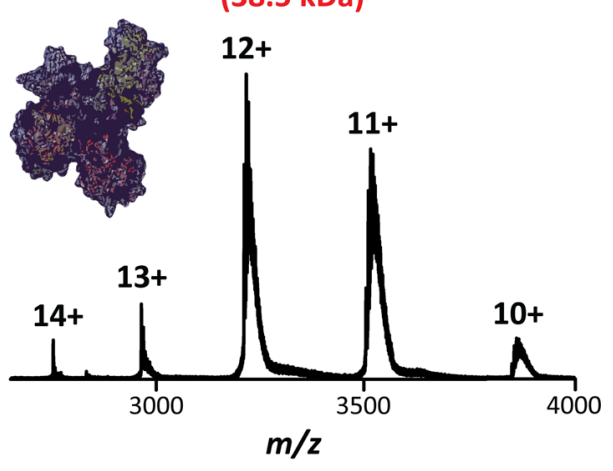

b) $\quad 13456789$

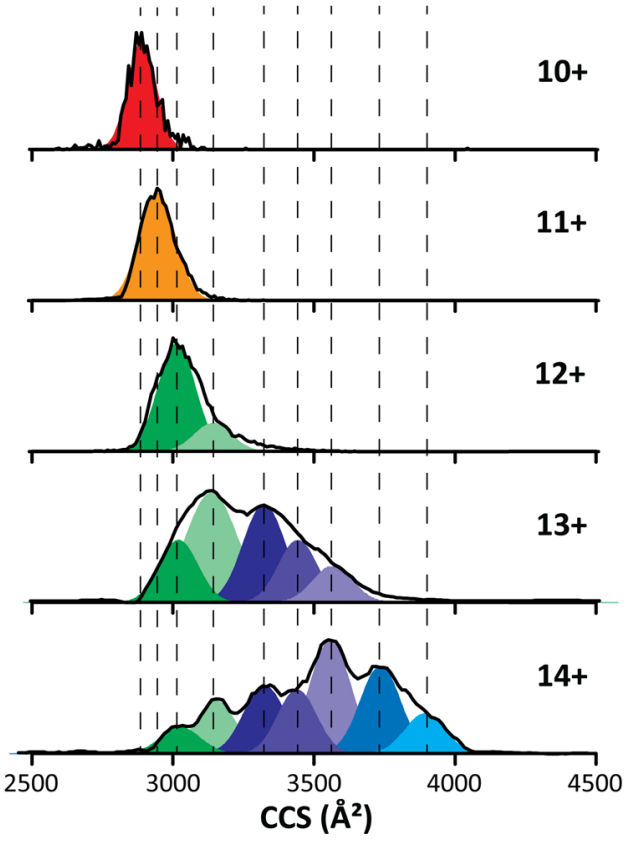

Figure 4. Typical (a) MS and (b) TIMS spectra for the multiply protonated species of variola virus topoisomerase IB. The dashed lines highlight the different microstates observed. The three-dimensional structure of vTopIB (PDB 2H7G) ${ }^{48}$ is shown in the inset for illustrative purposes.

of vTopIB. Inspection of the crystal structures of vTopIB in the DNA-bound form shows that a flexible linker connects the $\mathrm{N}$-and C-terminal domains, ${ }^{42,43}$ allowing both ends of the enzyme to surround the DNA substrate and form contacts on opposite sides of the duplex. ${ }^{44}$ This supports our findings on the number of transitional intermediates during the vTopIB free-rotation mechanism. In particular, our results suggest that movements of these domains away from each other can stem from Coulombic repulsion of exposed basic residues to protonation from these domains, which in the absence of DNA are exposed and not stabilized.

The interaction of TopIB with duplex DNA occurs via single-strand break to form a covalent intermediate bond between the catalytic tyrosine and the 3 '-phosphoryl end of the broken strand. ${ }^{44}$ The analysis of the variola virus topoisomerase IB in the DNA-bound form to a stem-loop DNA $(57 \mathrm{kDa})$ harboring the preferred binding sequence $5^{\prime}$-CCCTG-3 $3^{\prime 45-47}$ under native conditions exhibited a 1:1 binding stoichiometry across three charge states $(12+$ to $14+$, Figure 5a). Inspection 
vTopIB + Stem Loop DNA (57 kDa)
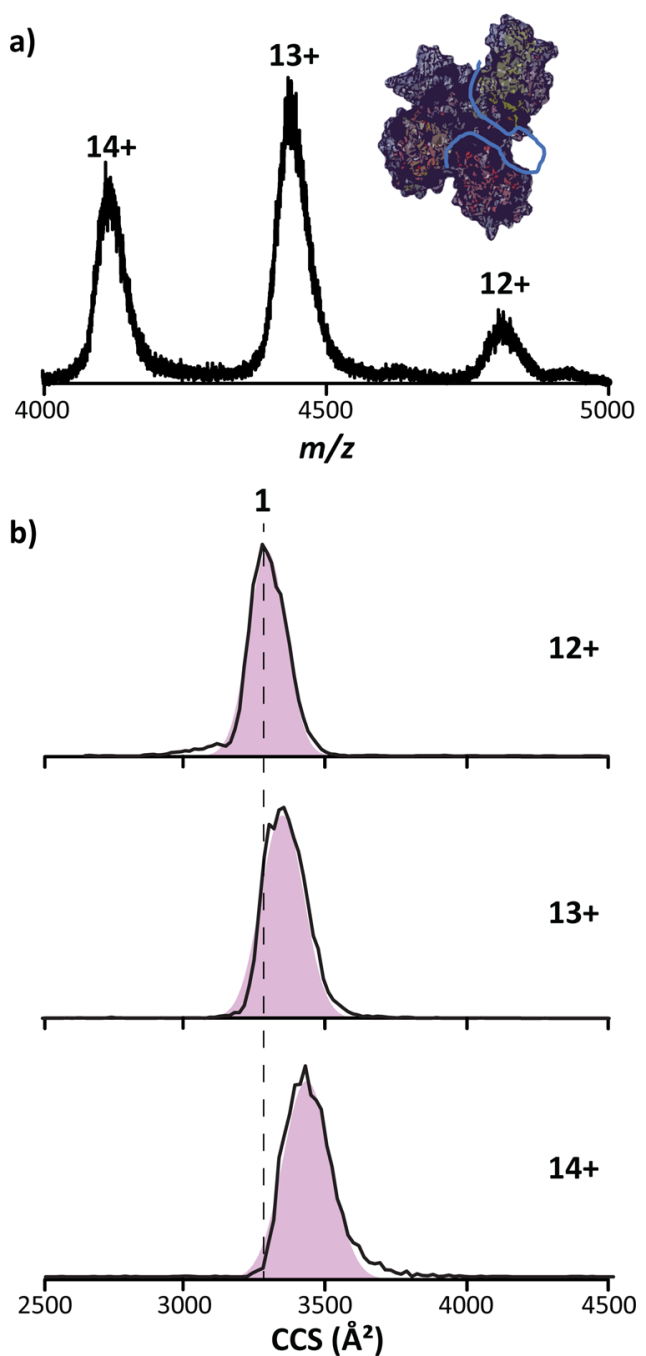

Figure 5. Typical (a) MS and (b) TIMS spectra for the multiply protonated species of variola virus topoisomerase IB in complex with a stem-loop DNA. The dashed lines highlight the different microstates observed. The three-dimensional structure of vTopIB DNA binding $(\mathrm{PDB} 2 \mathrm{H} 7 \mathrm{G})^{48}$ is shown in the inset for illustrative purposes.

of the IMS profiles displayed small changes in the CCS values when increasing the charge state, suggesting that the stem-loop DNA binding selects for a narrow range of conformers restricted by the interaction with the DNA substrate (Figure $5 b)$. Comparison of the CCS range between vTopIB and the DNA-bound form with a stem-loop DNA shows a much narrower CCS range for the DNA binding $\left(\sim 150 \AA^{2}\right.$ versus $\sim 1000 \AA^{2}$ ) across the same charge states. Inspection of the IMS profiles for the vTopIB DNA-bound form with a stem loop DNA shows narrower mobility bands at similar CCS as compared to the unbound vTopIB for the same charge states, resulting in the identification of only one microstate, which is slightly more elongated when increasing the charge state probably because of the Coulombic repulsion (Figure $5 \mathrm{~b}$ ). This trend suggests that the stem-loop DNA efficiently reduces the flexibility of the enzyme. In addition, comparison of the $+12,+13$, and +14 protonated species of both the unbound and DNA-bound enzyme revealed that the DNA complex achieves more compact conformations as compared to the denatured states of the unbound vTopIB (Table S2). Hence, the DNA stem loop induces the transition to a more compact structure. The formation of vTopIB into a folded C-shaped clamp, enveloping both the major and minor grooves of DNA, is strongly supported by this IMS results.

2.3. Comparison between EcTopIA and vTopIB Observations. The comparison of the charge state distributions and mobility profiles of the unbound EcTopIA and vTopIB shows some major differences, relating specifically to the disparity of each enzyme size $(97.5 \mathrm{kDa}$ vs $38.5 \mathrm{kDa}$, respectively). For example, closer investigation reveals that the unfolding of the native-like states of vTopIB is more easily induced in comparison to EcTopIA. Our results suggest that the greater conformational flexibility of the unbound vTopIB is most likely attributed to the motion of the interdomain hinge region ${ }^{49}$ or the protease-sensitive bridge, ${ }^{50}$ and that a strong intramolecular interaction network is present in EcTopIA that stabilizes the enzyme in a compact form. Comparison of the DNA-bound states shows similar trends despite inherent differences on the charge state distributions, CCS profiles, and number of conformational space. For example, for both enzymes, the binding to DNA significantly reduces their flexibility.

\section{CONCLUSIONS}

A detailed investigation of the microheterogeneity of EcTopIA and vTopIB in their unbound DNA-bound states is presented using a second-generation TIMS-MS. In particular, the analytical power of native TIMS-MS for the study of intrinsically disordered and dynamic enzymes and their complexes is shown. The EcTopIA and vTopIB enzymes exhibited differences in their intermediates. The unfolding of vTopIB is more easily induced, whereas the compact structures of EcTopIA evidence the presence of a strong intramolecular interaction network. The present results revealed that the DNA binding plays a significant role in reducing the flexibility of the topoisomerases. A number of microstates are reported for the unbound EcTopIA and vTopIB enzymes, as well as for the DNA-bound forms to single-stranded DNA and to a stem-loop DNA. CCS values are reported for all the microstates, which will further enable the generation of candidate three dimensional structures.

This work highlights the unique advantages of native TIMS-MS compared to traditional techniques with respect to the small amount of material needed for the analysis and the analysis time (millisecond timescale). For the case of the structural characterization of enzyme-DNA complexes, the TIMS-MS technology provides unique experimental findings to better understand the mechanisms involved in DNA substrate recognition, binding, and assembly of the catalytically active enzyme-DNA complex.

\section{EXPERIMENTAL SECTION}

4.1. Materials and Reagents. The E. coli topoisomerase IA (97.5 kDa, sequence: UniProt P06612) was expressed and purified as described elsewhere. ${ }^{10,51}$ The variola virus topoisomerase IB (38.5 kDa, sequence: UniProt P68697) was expressed and purified as described previously. ${ }^{52}$ Singlestranded DNA (5'-AATGCGCTTTGGG-3') with the preferred cleavage site of EcTopIA used previously in structural studies $^{53}$ and stem loop DNA (5'-GCCCTGAAAGATTATGGAA T G C GA T T A G G G T - AAA G GAA GA GA G CATAATCTTTCAGGGC-3') for characterizing EcTopIA activ- 
ity, ${ }^{54-57}$ consisting of a 16-base-pair stem and a 27-base loop, were provided by Biosearch Technologies (Petaluma, CA) and dialyzed in $100 \mathrm{mM}$ aqueous ammonium acetate $\left(\mathrm{NH}_{4} \mathrm{Ac}\right)$ before analysis. Topoisomerases were prepared at a final concentration of $10 \mu \mathrm{M}$ in $100 \mathrm{mM}$ aqueous $\mathrm{NH}_{4} \mathrm{Ac}$ under native conditions $(\mathrm{pH}=6.7)$. For DNA complexes, a $1: 1$ ratio of $5 \mu \mathrm{M}$ DNA and enzyme was prepared in $100 \mathrm{mM}$ aqueous $\mathrm{NH}_{4} \mathrm{Ac}$ immediately prior nESI infusion. Low-concentration Tuning Mix calibration standard (TuneMix, G24221A) was purchased from Agilent Technologies (Santa Clara, CA) and used to calibrate the instrument.

4.2. Native TIMS-MS Experiments. Ion mobility experiments were performed on a custom-built nanoESI-TIMS coupled to an Impact Q-TOF mass spectrometer (Bruker, Billerica, MA, Figure S4). ${ }^{15}$ Different from previous studies, a second-generation convex TIMS geometry was utilized to increase the trapping efficiency, resolving power, and the $\mathrm{m} / \mathrm{z}$ range. ${ }^{58}$ The TIMS unit is run by custom software in LabView (National Instruments) synchronized with the MS platform controls. ${ }^{31}$ Sample aliquots $(10 \mu \mathrm{L})$ were loaded in a pulled-tip capillary (O.D.: $1.0 \mathrm{~mm}$ and I.D.: $0.70 \mathrm{~mm}$ ) and sprayed at 600-1500 V. Briefly, the ion mobility separation in a TIMS device is based on holding the ions stationary using an electric field (E) against a moving buffer gas (Figure S4). ${ }^{59}$ TIMS separation depends on the gas flow velocity $\left(v_{\mathrm{g}}\right)$, elution voltage $\left(V_{\text {elution }}\right)$, ramp time $\left(t_{\text {ramp }}\right)$, and base voltage $\left(V_{\text {out }}\right){ }^{15,59}$ The reduced mobility, $K_{0}$, is defined by

$$
K_{0}=\frac{v_{\mathrm{g}}}{E} \cong \frac{A}{\left(V_{\text {elution }}-V_{\text {out }}\right)}
$$

The constant $A$ is determined using calibration standards (Tuning Mix) of known reduced mobilities. ${ }^{60}$ In TIMS operation, multiple conformers are trapped simultaneously at different $E$ values resulting from a voltage gradient applied across the IMS tunnel region (Figure S4). After thermalization, conformers are eluted by decreasing the electric field in stepwise decrements (Figure S4). Each conformer eluting from the TIMS cell can be described by a characteristic voltage $\left(V_{\text {elution }}\right)$. In a TIMS device, the total analysis time $\left(t_{\mathrm{T}}\right)$ can be described as

$$
t_{\mathrm{T}}=t_{\text {trap }}+\left(\frac{V_{\text {elution }}}{V_{\text {ramp }}}\right) t_{\text {ramp }}+\text { tof }
$$

where $t_{\text {trap }}$ is the thermalization/trapping time, tof is the time after the ion mobility separation, and $V_{\text {ramp }}$ and $t_{\text {ramp }}$ are the voltage range and time required to vary the electric field, respectively. The elution voltage is experimentally determined by varying the ramp time for a constant ramp voltage. A linear dependence of $t_{\mathrm{T}}$ on $t_{\text {ramp }}$ for all the investigated $\mathrm{m} / \mathrm{z}$ was obtained as describe elsewhere. ${ }^{60}$ From the slope and the intercept of this graph, the mobility values can be determined using the calibration constant A determined using known mobilities. Mobilities are converted into collision cross sections (CCS, $\AA^{2}$ ) using the Mason-Schamp equation

$$
\Omega=\frac{(18 \pi)^{1 / 2}}{16} \frac{q}{\left(k_{\mathrm{B}} T\right)^{1 / 2}}\left(\frac{1}{m}+\frac{1}{M}\right)^{1 / 2} \frac{1}{N} \times \frac{1}{K}
$$

where $q$ is the ion charge, $k_{\mathrm{B}}$ is the Boltzmann constant, $N$ is the gas number density, $m$ is the ion mass, and $M$ is the gas molecule mass. ${ }^{593}$ TIMS separation was carried out using nitrogen $\left(\mathrm{N}_{2}\right)$ at ambient temperature $(T)$ with $v_{\mathrm{g}}$ set by the pressure difference between the funnel entrance $(P 1=2.6$ mbar $)$ and exit $(P 2=1.1 \mathrm{mbar}$, Figure $\mathrm{S} 4)$. An rf voltage of $275 \mathrm{~V}_{\mathrm{pp}}$ at $880 \mathrm{kHz}$ was applied to all electrodes.

4.3. Data Processing. TIMS profiles were processed using a custom-built software-assisted molecular elucidation (SAME) package - a specifically designed two-dimensional (2D) TIMS-MS data processing script written in Python version 2.7. ${ }^{23,61}$ The SAME package utilizes noise removal, mean gap filling, "asymmetric least-squares smoothing" baseline correction, peak detection by a continuous wavelet transform-based peak detection algorithm (SciPy package), and Gaussian fitting with nonlinear least-squares functions (Levenberg-Marquardt algorithm). This procedure has been previously utilized for unsupervised mobility assignments from a complex mixture ${ }^{23,61}$ and is now presented for the case of proteins. To determine the number of IMS bands and to fit the IMS profiles, the narrowest observed peak for each molecular system was considered in the fitting criterion, leaving the centroid, area, and number of peaks as fitting parameters. The same fitting criterion can be applied to all charge states because the diffusion of the ions is negligible in a TIMS device, in contrast to other IMS technologies. The FWHM information per CCS is listed in Tables S1 and S2 for EcTopIA and vTopIB, respectively.

\section{ASSOCIATED CONTENT}

\section{Supporting Information}

The Supporting Information is available free of charge on the ACS Publications website at DOI: 10.1021/acsomega.8b02887.

Additional figures illustrating the three-dimensional structures of EcTopIA and vTopIB, TIMS-MS instrument showing the TIMS cell schematic and TIMS operation, and tables of the measured CCS and FWHM for all the investigated multiply protonated species of EcTopIA and vTopIB (PDF)

\section{AUTHOR INFORMATION}

\section{Corresponding Author}

*E-mail: fernandf@fiu.edu.

\section{ORCID}

Francisco Fernandez-Lima: 0000-0002-1283-4390

\section{Author Contributions}

$\mathrm{KJDF}$ and AL contributed equally to this work. The manuscript was written through contributions of all authors. All authors have given approval to the final version of the manuscript.

\section{Notes}

The authors declare no competing financial interest.

\section{ACKNOWLEDGMENTS}

The authors acknowledge the financial support from the National Science Foundation Division of Chemistry, under CAREER award CHE-1654274, with co-funding from the Division of Molecular and Cellular Biosciences to FFL, NIH grant R01GM054226 to YT, and NIAID grant 1R21AI125973 to FL. 


\section{REFERENCES}

(1) Vos, S. M.; Tretter, E. M.; Schmidt, B. H.; Berger, J. M. All tangled up: how cells direct, manage and exploit topoisomerase function. Nat. Rev. Mol. Cell Biol. 2011, 12, 827-841.

(2) Wang, J. C. Cellular roles of DNA topoisomerases: a molecular perspective. Nat. Rev. Mol. Cell Biol. 2002, 3, 430-440.

(3) Chen, S. H.; Chan, N. L.; Hsieh, T. S. New mechanistic and functional insights into DNA topoisomerases. Annu. Rev. Biochem. 2013, 82, 139-170.

(4) Tse-Dinh, Y. C. Bacterial topoisomerase I as a target for discovery of antibacterial compounds. Nucleic Acids Res. 2009, 37, 731-737.

(5) Peter, B. J.; Ullsperger, C.; Hiasa, H.; Marians, K. J.; Cozzarelli, N. R. The Structure of Supercoiled Intermediates in DNA Replication. Cell 1998, 94, 819-827.

(6) Dill, K. A.; MacCallum, J. L. The protein-folding problem, 50 years on. Science 2012, 338, 1042-1046.

(7) Yu, L.; Zhu, C. X.; Tse-Dinh, Y. C.; Fesik, S. W. Backbone dynamics of the C-terminal domain of Escherichia coli topoisomerase I in the absence and presence of single-stranded DNA. Biochemistry 1996, 35, 9661-9666.

(8) Kwon, K.; Jiang, Y. L.; Song, F.; Stivers, J. T. 19F NMR studies of vaccinia type IB topoisomerase. Conformational dynamics of the bound DNA substrate. J. Biol. Chem. 2002, 277, 353-358.

(9) Changela, A.; DiGate, R. J.; Mondragon, A. Crystal structure of a complex of a type IA DNA topoisomerase with a single-stranded DNA molecule. Nature 2001, 411, 1077-1081.

(10) Tan, K.; Zhou, Q.; Cheng, B.; Zhang, Z.; Joachimiak, A.; TseDinh, Y. C. Structural basis for suppression of hypernegative DNA supercoiling by E. coli topoisomerase I. Nucleic Acids Res. 2015, 43, 11031-11046.

(11) Pierson, N. A.; Chen, L.; Valentine, S. J.; Russell, D. H.; Clemmer, D. E. Number of solution states of bradykinin from ion mobility and mass spectrometry measurements. J. Am. Chem. Soc. 2011, 133, 13810-13813.

(12) Beveridge, R.; Chappuis, Q.; Macphee, C.; Barran, P. Mass spectrometry methods for intrinsically disordered proteins. Analyst 2013, 138, 32-42.

(13) Ruotolo, B. T.; Hyung, S. J.; Robinson, P. M.; Giles, K.; Bateman, R. H.; Robinson, C. V. Ion mobility-mass spectrometry reveals long-lived, unfolded intermediates in the dissociation of protein complexes. Angew. Chem. Int. Ed. 2007, 46, 8001-8004.

(14) Knapman, T. W.; Morton, V. L.; Stonehouse, N. J.; Stockley, P. G.; Ashcroft, A. E. Determining the topology of virus assembly intermediates using ion mobility spectrometry-mass spectrometry. Rapid Commun. Mass Spectrom. 2010, 24, 3033-3042.

(15) Fernandez-Lima, F.; Kaplan, D. A.; Suetering, J.; Park, M. A. Gas-phase separation using a Trapped Ion Mobility Spectrometer. Int. J. Ion Mobil. Spectrom. 2011, 14, 93-98.

(16) Fernandez-Lima, F. A.; Kaplan, D. A.; Park, M. A. Note: Integration of trapped ion mobility spectrometry with mass spectrometry. Rev. Sci. Instrum. 2011, 82, 126106.

(17) Silveira, J. A.; Ridgeway, M. E.; Park, M. A. High resolution trapped ion mobility spectrometery of peptides. Anal. Chem. 2014, 86, 5624-5627.

(18) Pu, Y.; Ridgeway, M. E.; Glaskin, R. S.; Park, M. A.; Costello, C. E.; Lin, C. Separation and Identification of Isomeric Glycans by Selected Accumulation-Trapped Ion Mobility Spectrometry-Electron Activated Dissociation Tandem Mass Spectrometry. Anal. Chem. 2016, 88, 3440-3443.

(19) Jeane Dit Fouque, K.; Moreno, J.; Hegemann, J. D.; Zirah, S.; Rebuffat, S.; Fernandez-Lima, F. Identification of Lasso Peptide Topologies Using Native Nanoelectrospray Ionization-Trapped Ion Mobility Spectrometry-Mass Spectrometry. Anal. Chem. 2018, 90, $5139-5146$

(20) Garabedian, A.; Baird, M. A.; Porter, J.; Jeanne Dit Fouque, K.; Shliaha, P. V.; Jensen, O. N.; Williams, T. D.; Fernandez-Lima, F.; Shvartsburg, A. A. Linear and Differential Ion Mobility Separations of Middle-Down Proteoforms. Anal. Chem. 2018, 90, 2918-2925.
(21) Jeanne Dit Fouque, K.; Garabedian, A.; Porter, J.; Baird, M.; Pang, X.; Williams, T. D.; Li, L.; Shvartsburg, A.; Fernandez-Lima, F. Fast and Effective Ion Mobility-Mass Spectrometry Separation of dAmino-Acid-Containing Peptides. Anal. Chem. 2017, 89, 1178711794.

(22) Adams, K. J.; Montero, D.; Aga, D.; Fernandez-Lima, F. Isomer Separation of Polybrominated Diphenyl Ether Metabolites using nanoESI-TIMS-MS. Int. J. Ion Mobil. Spectrom. 2016, 19, 69-76.

(23) Benigni, P.; Sandoval, K.; Thompson, C. J.; Ridgeway, M. E.; Park, M. A.; Gardinali, P.; Fernandez-Lima, F. Analysis of Photoirradiated Water Accommodated Fractions of Crude Oils Using Tandem TIMS and FT-ICR MS. Environ. Sci. Technol. 2017, 51, 5978-5988.

(24) Benigni, P.; Thompson, C. J.; Ridgeway, M. E.; Park, M. A.; Fernandez-Lima, F. Targeted high-resolution ion mobility separation coupled to ultrahigh-resolution mass spectrometry of endocrine disruptors in complex mixtures. Anal. Chem. 2015, 87, 4321-4325.

(25) Ridgeway, M. E.; Silveira, J. A.; Meier, J. E.; Park, M. A. Microheterogeneity within conformational states of ubiquitin revealed by high resolution trapped ion mobility spectrometry. Analyst 2015, 140, 6964-6972.

(26) Jeanne Dit Fouque, K.; Salgueiro, L. M.; Cai, R.; Sha, W.; Schally, A. V.; Fernandez-Lima, F. Structural Motif Descriptors as a Way To Elucidate the Agonistic or Antagonistic Activity of Growth Hormone-Releasing Hormone Peptide Analogues. ACS Omega 2018, 3, 7432-7440.

(27) Schenk, E. R.; Ridgeway, M. E.; Park, M. A.; Leng, F.; Fernandez-Lima, F. Isomerization Kinetics of AT Hook Decapeptide Solution Structures. Anal. Chem. 2014, 86, 1210-1214.

(28) Garabedian, A.; Leng, F.; Ridgeway, M. E.; Park, M. A.; Fernandez-Lima, F. Tailoring peptide conformational space with organic gas modifiers in TIMS-MS. Int. J. Ion Mobil. Spectrom. 2018, $21,43-48$

(29) Liu, F. C.; Kirk, S. R.; Bleiholder, C. On the structural denaturation of biological analytes in trapped ion mobility spectrometry - mass spectrometry. Analyst 2016, 141, 3722-3730.

(30) Garabedian, A.; Butcher, D.; Lippens, J. L.; Miksovska, J.; Chapagain, P. P.; Fabris, D.; Ridgeway, M. E.; Park, M. A.; FernandezLima, F. Structures of the kinetically trapped i-motif DNA intermediates. Phys. Chem. Chem. Phys. 2016, 18, 26691-26702.

(31) Butcher, D.; Chapagain, P.; Leng, F.; Fernandez-Lima, F. Differentiating Parallel and Antiparallel DNA Duplexes in the Gas Phase Using Trapped Ion Mobility Spectrometry. J. Phys. Chem. B 2018, 122, 6855-6861.

(32) Molano-Arevalo, J. C.; Jeanne Dit Fouque, K.; Pham, K.; Miksovska, J.; Ridgeway, M. E.; Park, M. A.; Fernandez-Lima, F. Characterization of Intramolecular Interactions of Cytochrome $c$ Using Hydrogen-Deuterium Exchange-Trapped Ion Mobility Spectrometry-Mass Spectrometry and Molecular Dynamics. Anal. Chem. 2017, 89, 8757-8765.

(33) Schenk, E. R.; Almeida, R.; Miksovska, J.; Ridgeway, M. E.; Park, M. A.; Fernandez-Lima, F. Kinetic Intermediates of Holo- and Apo-Myoglobin Studied Using HDX-TIMS-MS and Molecular Dynamic Simulations. J. Am. Soc. Mass Spectrom. 2015, 26, 555-563.

(34) Garabedian, A.; Bolufer, A.; Leng, F.; Fernandez-Lima, F. Peptide Sequence Influence on the Conformational Dynamics and DNA binding of the Intrinsically Disordered AT-Hook 3 Peptide. Sci. Rep. 2018, 8, 10783.

(35) Dekker, N. H.; Rybenkov, V. V.; Duguet, M.; Crisona, N. J.; Cozzarelli, N. R.; Bensimon, D.; Croquette, V. The mechanism of type IA topoisomerases. Proc. Natl. Acad. Sci. U.S.A. 2002, 99, 1212612131.

(36) Stivers, J. T.; Harris, T. K.; Mildvan, A. S. Vaccinia DNA topoisomerase I: evidence supporting a free rotation mechanism for DNA supercoil relaxation. Biochemistry 1997, 36, 5212-5222.

(37) Feinberg, H.; Changela, A.; Mondragon, A. Protein-nucleotide interactions in E. coli DNA topoisomerase I. Nat. Struct. Biol. 1999, 6, 961-968. 
(38) Feinberg, H.; Lima, C. D.; Mondragon, A. Conformational changes in E. coli DNA topoisomerase I. Nat. Struct. Biol. 1999, 6, 918-922.

(39) Sissi, C.; Cheng, B.; Lombardo, V.; Tse-Dinh, Y. C.; Palumbo, M. Metal ion and inter-domain interactions as functional networks in E. coli topoisomerase I. Gene 2013, 524, 253-260.

(40) Perry, K.; Mondragon, A. Biochemical characterization of an invariant histidine involved in Escherichia coli DNA topoisomerase I catalysis. J. Biol. Chem. 2002, 277, 13237-13245.

(41) Mills, M.; Tse-Dinh, Y. C.; Neuman, K. C. Direct observation of topoisomerase IA gate dynamics. Nat. Struct. Mol. Biol. 2018, 25, $1111-1118$.

(42) Perry, K.; Hwang, Y.; Bushman, F. D.; Van Duyne, G. D. Insights from the structure of a smallpox virus topoisomerase-DNA transition state mimic. Structure 2010, 18, 127-137.

(43) Patel, A.; Yakovleva, L.; Shuman, S.; Mondragon, A. Crystal structure of a bacterial topoisomerase IB in complex with DNA reveals a secondary DNA binding site. Structure 2010, 18, 725-733.

(44) Koster, D. A.; Croquette, V.; Dekker, C.; Shuman, S.; Dekker, $\mathrm{N}$. H. Friction and torque govern the relaxation of DNA supercoils by eukaryotic topoisomerase IB. Nature 2005, 434, 671-674.

(45) Shuman, S. Site-specific interaction of vaccinia virus topoisomerase I with duplex DNA. Minimal DNA substrate for strand cleavage in vitro. J. Biol. Chem. 1991, 266, 11372-11379.

(46) Shuman, S. Site-specific DNA cleavage by vaccinia virus DNA topoisomerase I. Role of nucleotide sequence and DNA secondary structure. J. Biol. Chem. 1991, 266, 1796-1803.

(47) Shuman, S. Recombination mediated by vaccinia virus DNA topoisomerase I in Escherichia coli is sequence specific. Proc. Natl. Acad. Sci. U.S.A. 1991, 88, 10104-10108.

(48) Perry, K.; Hwang, Y.; Bushman, F. D.; Van Duyne, G. D. Structural basis for specificity in the poxvirus topoisomerase. Mol. Cell 2006, 23, 343-354.

(49) Sekiguchi, J.; Shuman, S. Proteolytic footprinting of vaccinia topoisomerase bound to DNA. J. Biol. Chem. 1995, 270, 1163611645 .

(50) Cheng, C.; Kussie, P.; Pavletich, N.; Shuman, S. Conservation of Structure and Mechanism between Eukaryotic Topoisomerase I and Site-Specific Recombinases. Cell 1998, 92, 841-850.

(51) Xu, X.; Leng, F. A rapid procedure to purify Escherichia coli DNA topoisomerase I. Protein Expr. Purif. 2011, 77, 214-219.

(52) Ahmad, M.; Xue, Y.; Lee, S. K.; Martindale, J. L.; Shen, W.; Li, W.; Zou, S.; Ciaramella, M.; Debat, H.; Nadal, M.; Leng, F.; Zhang, H.; Wang, Q.; Siaw, G. E.; Niu, H.; Pommier, Y.; Gorospe, M.; Hsieh, T. S.; Tse-Dinh, Y. C.; Xu, D.; Wang, W. RNA topoisomerase is prevalent in all domains of life and associates with polyribosomes in animals. Nucleic Acids Res. 2016, 44, 6335-6349.

(53) Zhang, Z.; Cheng, B.; Tse-Dinh, Y. C. Crystal structure of a covalent intermediate in DNA cleavage and rejoining by Escherichia coli DNA topoisomerase I. Proc. Natl. Acad. Sci. U.S.A. 2011, 108, 6939-6944.

(54) Narula, G.; Annamalai, T.; Aedo, S.; Cheng, B.; Sorokin, E.; Wong, A.; Tse-Dinh, Y. C. The strictly conserved Arg-321 residue in the active site of Escherichia coli topoisomerase I plays a critical role in DNA rejoining. J. Biol. Chem. 2011, 286, 18673-18680.

(55) Narula, G.; Tse-Dinh, Y. C. Residues of E. coli topoisomerase I conserved for interaction with a specific cytosine base to facilitate DNA cleavage. Nucleic Acids Res. 2012, 40, 9233-9243.

(56) Cheng, B.; Annamalai, T.; Sorokin, E.; Abrenica, M.; Aedo, S.; Tse-Dinh, Y. C. Asp-to-Asn substitution at the first position of the DxD TOPRIM motif of recombinant bacterial topoisomerase $\mathrm{I}$ is extremely lethal to E. coli. J. Mol. Biol. 2009, 385, 558-567.

(57) Cheng, B.; Sorokin, E. P.; Tse-Dinh, Y. C. Mutation adjacent to the active site tyrosine can enhance DNA cleavage and cell killing by the TOPRIM Gly to Ser mutant of bacterial topoisomerase I. Nucleic Acids Res. 2008, 36, 1017-1025.

(58) Jeanne Dit Fouque, K.; Garabedian, A.; Leng, F.; Tse-Dinh, Y. C.; Koleva, B. N.; Beuning, P. J.; Ridgeway, M.; Park, M.; FernandezLima, F. High-Resolution Native Ion Mobility - Mass Spectrometry
Analysis of Intact Macromolecular Assemblies. Anal. Chem. 2018, submitted.

(59) McDaniel, E. W.; Mason, E. A. The Mobility and diffusion of ions in gases. John Wiley and Sons, Inc. , New York: New York, 1973; p 381.

(60) Hernandez, D. R.; Debord, J. D.; Ridgeway, M. E.; Kaplan, D. A.; Park, M. A.; Fernandez-Lima, F. Ion dynamics in a trapped ion mobility spectrometer. Analyst 2014, 139, 1913-1921.

(61) Tose, L. V.; Benigni, P.; Leyva, D.; Sundberg, A.; Ramirez, C. E.; Ridgeway, M. E.; Park, M. A.; Romao, W.; Jaffe, R.; FernandezLima, F. Coupling trapped ion mobility spectrometry to mass spectrometry: trapped ion mobility spectrometry-time-of-flight mass spectrometry versus trapped ion mobility spectrometry-Fourier transform ion cyclotron resonance mass spectrometry. Rapid Commun. Mass Spectrom. 2018, 32, 1287-1295. 